\title{
Minute Times Micromole per Liter per Meter Squared
}

National Cancer Institute

\section{Source}

National Cancer Institute. Minute Times Micromole per Liter per Meter Squared. NCI

Thesaurus. Code C111261.

Minutes times micromoles per liter, divided by meters squared. 